\title{
Two-gene mutation in a single patient: Biochemical and functional analysis for a correct interpretation of exome results
}

\author{
ANNA MONICA BIANCO, FLAVIO FALETRA, DIEGO VOZZI, MARTINA GIRARDELLI, \\ ALESSANDRA KNOWLES, ALBERTO TOMMASINI, GIORGIO ZAULI and ANNALISA MARCUZZI \\ Department of Advanced Diagnostic and Clinical Trials, \\ Institute for Maternal and Child Health - IRCCS 'Burlo Garofolo', Trieste, Trieste 34137, Italy
}

Received December 2, 2014; Accepted June 23, 2015

DOI: $10.3892 / \mathrm{mmr} .2015 .4215$

\begin{abstract}
Next-generation sequencing (NGS) has generated a large amount of sequence data with the requirement of frequent critical revisions of reported mutations. This innovative tool has proved to be effective in detecting pathogenic mutations; however, it requires a certain degree of experience to identify incidental findings. In the present study, whole exome sequencing analysis was performed for the molecular diagnosis and correct genotype/phenotype correlation between parents and a patient presenting with an atypical phenotype. In addition, mevalonic acid quantification and frequency analysis of detected variants in public databases and X-chromosome inactivation (XCI) studies on the patient's mother were performed. V377I as well as the S135L mutations were identified on the mevalonate kinase deficiency gene and the levels of mevalonic acid in the patient were 5,496 $\mu \mathrm{g} / \mathrm{ml}$. A D59G variation, reported in ESP6500 in two healthy individuals, was found on the Martin Probst syndrome gene (RAB40AL). Based on XCI studies on the patient's mother, it is likely that $R A B 40 A L$ escapes $\mathrm{XCI}$, while still remaining balanced. In conclusion, the results of the present study indicated that the Martin Probst syndrome is an X-linked condition, which is probably not caused by $R A B 40 A L$ mutations. Although NGS is a powerful tool to identify pathogenic mutations, the analysis of genetic data requires expert critical revision of all detected variants.
\end{abstract}

\section{Introduction}

Next-generation sequencing (NGS) has introduced a novel era, in which it has become possible to rapidly and

Correspondence to: Dr Annalisa Marcuzzi, Department of Advanced Diagnostic and Clinical Trials, Institute for Maternal and Child Health - IRCCS ‘Burlo Garofolo', 65/1 Via dell' Istria, Trieste, Trieste 34137, Italy

E-mail: annalisa.marcuzzi@burlo.trieste.it

Key words: mevalonate, rare disease, RAB40AL, mevalonate kinase deficiency, genotype-phenotype cost-efficiently obtain large amounts of sequence data for patients. NGS analysis is therefore currently regarded as a first-choice diagnostic tool, particularly in cases in which phenotypical and clinical features are unclear or atypical, leaving Sanger sequencing for the confirmation of detected variants (1). The correct placement of a preliminary clinical diagnosis and subsequent selection of candidate genes for molecular analysis are challenging when patients present with signs and symptoms that do not match any known clinical condition. In these cases, the use of Sanger sequencing to produce a molecular diagnosis is unreasonable due to the high cost and effort required.

In these cases, whole exome sequencing (WES) can be an appropriate tool to detect pathogenic mutations; however, this requires an effective strategy for filtering results and in-depth analysis to meaningfully interpret incidental findings $(2,3)$. Furthermore, in silico and functional studies may be necessary to attribute the correct value to unexpected results.

The present study reports on a patient who was selected, given the clinical phenotype complexity, for proband-parents (trios) analysis using WES with the aim of detecting all the possible genetic variants that may have generated the patient's phenotype.

The present study confirmed that it is essential to filter and confirm each step of the NGS based on the decision of a team that has the necessary expertise to assess the impact of the detected variants.

\section{Materials and methods}

Ethical approval. The technical and scientific review board of the Institute for Maternal and Child Health, Istituto di Ricovero e Cura a Carattere Scientifico (IRCCS) 'Burlo Garofolo' (Trieste, Italy) (no. 185/08, 19/08/2008) approved the present study. For enrolment of the child and the parents in the present study, informed consent was obtained from the parents.

Patient. A six-year-old boy with non-consanguineous parents was admitted to the Paediatric Department of the IRCCS 'Burlo Garofolo' (Trieste, Italy) due to neurogenic diabetes insipidus, mild hepatomegaly and recurrent episodes of fever with skin rash and cervical adenitis. 
DNA extraction. Genomic DNA (gDNA) was extracted from 1-2 ml EDTA-anticoagulated blood obtained from the proband and his parents using an EZ1 DNA Blood kit (Qiagen, Hilden, Germany) according to manufacturer's instructions.

WES analysis. A total of $3 \mu \mathrm{g}$ gDNA was used for library preparation using TruSeq ${ }^{\mathrm{TM}}$ Exome Enrichment $62 \mathrm{Mb}$ kit (Illumina Inc., San Diego, CA, USA). A paired-end sequencing of each library was performed on an Illumina HiSeq1000 (Illumina Inc.). Raw sequencing data were collected as unmapped 100-bp reads in fastQ format. CLC Genomics Workbench version 6.5 software (CLC Bio; Qiagen) was employed to check the quality of the reads, to map reads back to the human reference genome, version hg19, to calculate the overall coverage, to perform local re-alignment and base quality re-calibration and, finally, to call single nucleotide variants (SNVs) and small insertion/deletions (INDELs). All of these data were collected into a standardized variant call format, version 4.1 (4). SNVs/INDELs were annotated using ANNOVAR software (http://www.openbioinformatics.org/annovar/; version 2013Aug23) referring to several public databases, including National Center of Biotechnology Information dbSNP build138 (http://www.ncbi.nlm.nih.gov/SNP/), the 1000 Genomes Project (http://www.1000genomes.org/) and the National Heart, Lung, and Blood Institute Exome Sequencing Project Exome Variant Server (http://evs.gs.washington. edu/EVS/) (5).

Bioinformatic and statistical analyses. SNVs/INDELs were first analyzed with the aim of evaluating all known mutations already associated with the clinical manifestations of the patient. Subsequently, SNVs/INDELs were filtered with the purpose of selecting variants which are poorly represented within the general population and potentially 'pathogenic'; to this end, variants were selected according to the following inclusion criteria: A) Minor allelic frequency $<0.03$ in residents with ancestry from northern and western Europe, referring to the 1000 Genomes Project (http://www.1000genomes.rg/) and NHLBI Exome Sequencing Project (ESP) Exome Variant Server (http://evs.gs.washington.du/EVS/); B) homozygous variants carried by the proband; and C) variants predicted as 'pathogenic' based on Polyphen-2 (6), Mutation Taster and LRT scores, as recorded in dbNSFP v2.0 $(7,8)$.

Polymerase chain reaction (PCR) and Sanger analyses. PCR amplification was performed for the specific gene-coding sequences of the $M V K$ and $R A B 40 A L$ genes. 50 ng DNA was amplified using KAPA 2G Fast Hot Start Readymix (initial concentration, $2 \mathrm{X}$; final concentration, $1 \mathrm{X}$; respective primer initial concentration, $10 \mu \mathrm{M}$; final concentration, $0.2 \mu \mathrm{M}$; final volume, $25 \mu \mathrm{l}$ ) (Resnova, Roma, Italy) and a 2720 Thermal Cycler (Applied Biosystems, Thermo Fisher Scientific, Waltham, MA, USA). Amplification products were purified with ExoSAP-IT enzyme (Affymetrix, Inc., Santa Clara, CA, USA) at $37^{\circ} \mathrm{C}$ for $20 \mathrm{sec}$, subsequently inactivated at $85^{\circ} \mathrm{C}$ for $10 \mathrm{sec}$, and directly sequenced by the Sanger method using ABI Prism 3.1 Big Dye terminator chemistry (Invitrogen Life Technologies, Carlsbad, CA, USA) and BigDye XTerminator Purification kit (Applied Biosystems). Runs were performed on the ABI Prism 3130XL automated DNA sequencer (Applied
Biosystems). Sequences were analyzed with Seqman II Software (DNASTAR I Lasergene 7.0; DNASTAR, Madison, WI, USA). PCR amplification and Sanger sequencing primers to confirm the $M V K$ (NM_000431) mutations (c.1129G>A; p.V377I) and (c.404C >T; p.S135L) were: $M V K 5$ forward, 5'-GTTGAGAAAACTGGACCAGATGC-3' and $M V K 5$ reverse, 5'-CTCAGCTTCCTCATGTTAAAATG-3'; and MVK11 forward, 5'-GGCTTTTGCCTTGAATATGATGA-3' and $M V K 11$ reverse, 5'-GGGCCTCTCCAGCAGTGTC-3'. PCR amplification and Sanger sequencing primers to confirm the RAB4OAL (NM_001031834) known dinucleotide missense changes c.A176G and c.C177A, leading to p.D59G were: $R A B 40 A L$ forward, 5'-CCTCTGCGCACAACCTTGC-3' and RAB40AL reverse, 5'- CTGGAGCGATTCCAGCTTG-3'. Primers were obtained from Integrated DNA Technologies (Coralville, IA, USA). PCR amplification cycle: Initial denaturation $\left(95^{\circ} \mathrm{C}\right.$ for $\left.1 \mathrm{sec}\right), 30$ cycles of denaturation $\left(95^{\circ} \mathrm{C}\right.$ for $10 \mathrm{sec})$, annealing $\left(60^{\circ} \mathrm{C}\right.$ for $\left.10 \mathrm{sec}\right)$ and elongation $\left(72^{\circ} \mathrm{C}\right.$ for $5 \mathrm{sec})$, followed by a final elongation $\left(72^{\circ} \mathrm{C}\right.$ for $\left.30 \mathrm{sec}\right)$. Sequence reaction cycle: Initial denaturation $\left(96^{\circ} \mathrm{C}\right.$ for $\left.3 \mathrm{sec}\right)$, 26 cycles of denaturation $\left(96^{\circ} \mathrm{C}\right.$ for $\left.30 \mathrm{sec}\right)$, annealing $\left(53^{\circ} \mathrm{C}\right.$ for $15 \mathrm{sec})$ and elongation $\left(60^{\circ} \mathrm{C}\right.$ for $\left.4 \mathrm{sec}\right)$.

RAB40AL inactivation analysis. $300 \mathrm{ng}$ genomic DNA of the patient was digested with $40 \mathrm{U}$ HpaII methylation-sensitive restriction enzyme and $20 \mathrm{U}$ RsaI methylation-insensitive restriction enzyme (New England Biolabs, Ipswich, MA, USA) in a total volume of $20 \mu \mathrm{l}$ for $24 \mathrm{~h}$ at $37^{\circ} \mathrm{C}$. After digestion, the mixtures were incubated at $95^{\circ} \mathrm{C}$ for $10 \mathrm{~min} .50 \mathrm{ng}$ digested and undigested DNA were amplified with $R A B 40 A L$ forward and reverse primers as previously described to verify the inactivation of the gene.

X-chromosome inactivation (XCI) analysis via androgen receptor. $300 \mathrm{ng}$ genomic DNA of the patient's mother was digested as described above and incubated at $95^{\circ} \mathrm{C}$ for $10 \mathrm{~min}$. $50 \mathrm{ng}$ of digested and undigested DNA was amplified with primers 5'-GCTGTGAAGGTTGCTGTTCCTCAT-3' and 5'-56-FAM/TCCAGAATCTGTTCCAGAGCGTGC-3' according to the protocol of a previous study (9) for amplification of a region of the human androgen receptor (HAR) gene, localized on Xq12 and characterized by a polymorphic number of CAG repeats. Since this fragment has two HpaII restriction enzyme sites, only methylated DNA was amplified.

XCI analysis via the polymorphic marker DXS1214. $300 \mathrm{ng}$ genomic DNA of the patient's mother was digested as described above and incubated at $95^{\circ} \mathrm{C}$ for $10 \mathrm{~min} .50 \mathrm{ng}$ digested and undigested DNA was amplified with specific DXS1214-labeled primers.

The labeled PCR products were denatured and run on the automated sequencer (ABI 3730XL Platform; Applied Biosystems) using the POP7 polymer and the ROX size standard as size markers (Invitrogen Life Technologies, Inc.). GeneMapper software (v. 4.0; Applied Biosystems) was used for fragment analysis.

Biochemical analysis. Urinary mevalonic acid was determined by means of gas chromatography/mass spectrometry. Spectra were obtained using a Hewlett Packard gas chromatograph 
Table I. Main symptoms for MP syndrome and MK deficiency compared with the symptoms of patient of the present study.

\begin{tabular}{|c|c|c|c|}
\hline Feature & MP syndrome & MK deficiency & Patient \\
\hline Growth & Short stature & $\begin{array}{l}\text { From failure to thrive }{ }^{\text {a }} \text { to normal; } \\
\text { hyper immunoglobulin D; } \\
\text { periodic fever syndrome }\end{array}$ & Normal \\
\hline Head & Microcephaly & Microcephaly $^{\mathrm{a}}$ & Normal \\
\hline Ears & Hearing loss & Normal & Normal \\
\hline Eye & Normal & Central cataracts ${ }^{\mathrm{a}}$ & Normal \\
\hline Dysmorphisms & $\begin{array}{l}\text { Low-set ears } \\
\text { Telecanthus } \\
\text { Hypertelorism } \\
\text { Epicanthal folds } \\
\text { Broad nasal root } \\
\text { Broad mouth } \\
\text { Full lower lip } \\
\text { Abnormal teeth }\end{array}$ & $\begin{array}{l}\text { Triangular face }{ }^{\mathrm{a}}, \\
\text { Hypoplastic alae nasi }^{\mathrm{a}}\end{array}$ & Normal \\
\hline Genitourinary & $\begin{array}{l}\text { Bifid scrotum } \\
\text { Small phallus } \\
\text { Cryptorchidism } \\
\text { Hypoplastic kidneys } \\
\text { Renal insufficiency }\end{array}$ & Normal & Normal \\
\hline Skin & Telangiectasias & Skin rashes & Skin rashes \\
\hline Central nervous system & $\begin{array}{l}\text { Mental retardation, } \\
\text { mild to severe }\end{array}$ & $\begin{array}{l}\text { Psychomotor retardation }{ }^{\mathrm{a}} \text {, } \\
\text { Cerebella ataxia }^{\mathrm{a}}\end{array}$ & $\begin{array}{l}\text { Neurogenic diabetes } \\
\text { insipidus }\end{array}$ \\
\hline Osteoarticular system & Normal & Arthralgia/arthritis & Normal \\
\hline Endocrine features & Hypothyroidism & Normal & Normal \\
\hline Hematology & Pancytopenia & Anemia & Mild anemia \\
\hline Lymphoid organs & Leukopenia & $\begin{array}{l}\text { Lymphadenopathy, } \\
\text { hepato-splenomegaly }\end{array}$ & $\begin{array}{l}\text { Lymphadenopathy, } \\
\text { hepato-splenomegaly }\end{array}$ \\
\hline Molecular Basis & $R A B 40 A L$ & $M V K$ & $\begin{array}{l}\text { MVK V377I/S135L } \\
\text { RAB40AL D59G }\end{array}$ \\
\hline
\end{tabular}

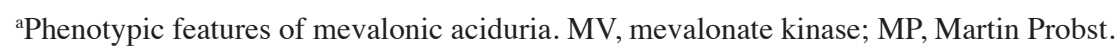

6890 series equipped with a Hewlett Packard 5973 quadrupole operating in electron-impact mode at $70 \mathrm{eV}$ (Hewlett Packard, Palo Alto, CA, USA) (10).

\section{Results and Discussion}

WES revealed two missense mutations of the $M V K$ gene (heterozygous): NM_000431, c.1129G>A; p.V377I (rs28934897); and NM-000431, c.404C>T; p.S135L (rs104895297). In addition, the known dinucleotide missense mutations of the $R A B 40 A L$ (NM_001031834) gene were detected: c. A176G (rs145606134) and c. C177A (rs138133927), leading to p.D59G. Sanger sequencing analysis confirmed all mutations. No mutations were detected in genes associated with diabetes insipidus.

The $M V K$ gene encodes mevalonate kinase, the third enzyme on the pathway leading to the synthesis of cholesterol from acetyl-CoA (11). Mutations of the $M V K$ gene cause a recessive inherited disease called mevalonate kinase deficiency (MKD), which manifests as a clinical and biochemical continuum with mevalonic aciduria at the most severe end [MA; online mendelian of inheritance in man (OMIM) \#610377] and hyper immunoglobulin D and periodic fever syndrome at the mildest end (HIDS; OMIM \#260920) (12).

The D59G variant of $R A B 40 A L$ has already been reported as causative of the Martin-Probst syndrome (MPS; OMIM \#300519), a recessive X-linked disease characterized by congenital sensorineural hearing loss, mental retardation, short stature, congenital umbilical hernia, facial dysmorphism, abnormal teeth, widely spaced nipples and abnormal dermatoglyphics $(13,14)$. In the present study, a multidisciplinary team discussed the relevance of the genetic results in order to compare the patient's clinical features with those pertaining to MKD and MPS, based on clinical, genetic and functional evaluations.

The diagnosis of MKD was supported not only by the fact that the two mutations detected have a well known role in the 
A
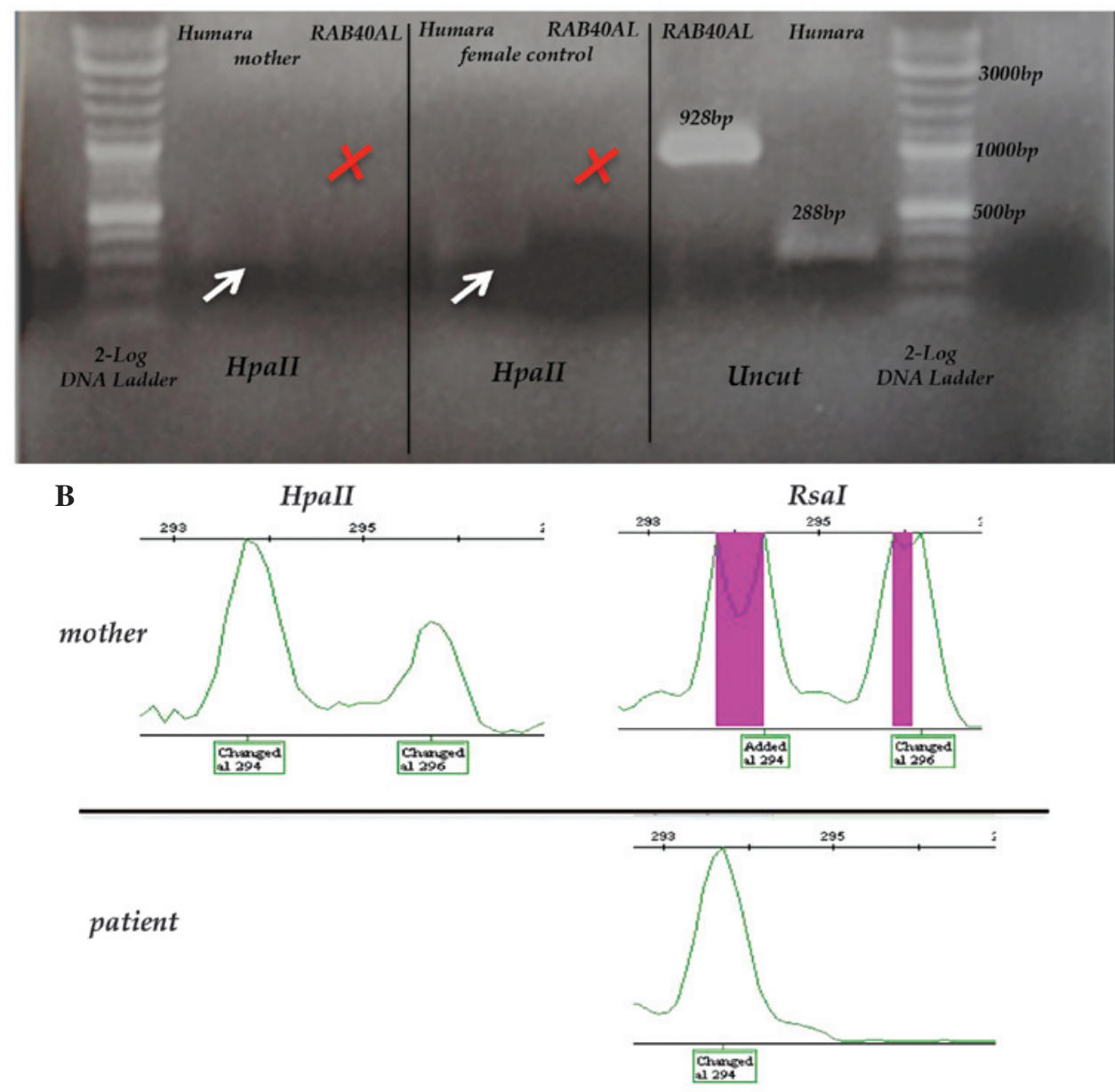

Figure 1. (A) Humara PCR results from digested genomic DNA ( HpaII) and uncut DNA. The RAB4OAL PCR product could only be amplified from undigested DNA. (B) Analysis of the DXS1214 polymorphic marker in genomic DNA digested with HpaII and in DNA digested with RsaI. The graphs suggest a balanced $\mathrm{X}$ inactivation. $\mathrm{PCR}$, polymerase chain reaction.

disease, but also by a biochemical analysis that demonstrated the accumulation of mevalonic acid [5,496 $\mu \mathrm{g} / \mathrm{ml}$; the normal range of mevalonic acid levels is $34-323 \mu \mathrm{g} / \mathrm{ml}$ (internal laboratory reference)] in urine during febrile crisis, as a proof of MK enzyme deficiency (10).

In the case of the present study, the diagnosis of MKD adequately explained the proband's condition, apart from the presence of neurogenic diabetes insipidus (Table I).

In fact, most of the clinical features of the patient are characteristics of MKD, although a large number of MKD-associated symptoms were not present in the patient. In particular, diabetes insipidus, which was one of the main complaints of the patient, is not characteristic of MKD. By contrast, no sign or symptom of MPS was present in the patient. Since MPS is described as an X-recessive disease with complete penetrance, it was unexpected that the patient had not developed any of the severe impairments, which are typical of the disease. It was thus hypothesized that either the defect in MK has a protective effect against the development of MPS or that the variant detected in $R A B 40 A L$ is not the actual cause of MPS. Of note, the variant identified in $R A B 40 A L$ was predicted to affect protein farnesylation; however, MKD is also associated with an abnormal pattern of protein prenylation due to a shortage of mevalonate-derived isoprenoids $(15,16)$. However, considering that the two defects can reduce protein farnesylation, the combined effect of the two defects would have been expected to be even more severe. On the contrary, the National Heart, Lung and Blood Institute-exome sequencing project (ESP)6500 database (http://evs.gs.washington.edu/EVS/) lists two healthy males carrying the $\mathrm{D} 59 \mathrm{G}$ variant of $R A B 40 A L$, suggesting that it represents an irrelevant albeit rare polymorphism. In addition, recent studies described the same $R A B 40 A L$ variant in a patient with a distinctly different phenotype from that reported in MPS patients (17,18).

To ascertain the possibility that $R A B 40 A L$ is not the causative gene of MPS, the present study exploited the opportunity of studying the mother of the patient, who is a heterozygous carrier of the same gene variant. Bedoyan et al (13) demonstrated that, while males affected by MPS develop progressive loss of white blood cells, female carriers have a normal leukocyte count and display a skewed XCI in peripheral blood cells. The present study then attempted to analyze the inactivation profile of $R A B 40 A L$ by amplifying the gene after digestion with a methylation-sensitive enzyme $(19,20)$. However, it was not possible to amplify RAB40AL after $H p a I I$ digestion, suggesting that this gene escapes inactivation (Fig. 1A). In order to verify the hypothesis of a skewed XCI, the present study then used polymorphic X-chromosome markers known to undergo methylation and inactivation. As the most widely used androgen receptor humara locus was uninformative in the patient's family, 
the polymorphic marker DXS1214 was then analyzed. After digestion with HpaII, which is selective for active unmethylated DNA, the two DXS1214 polymorphic alleles remained present to similar extents, confirming a balanced XCI (Fig. 1B). These results suggested that the pattern of skewed XCI in the family examined by Bedoyan et al (13) is not due to the D59G variant in $R A B 40 A L$, but to another unidentified $\mathrm{X}$-chromosome gene mutation. In conclusion, these results suggested that mutations of $R A B 40 A L$ are not the cause of MPS.

The case of the present study confirmed the potential of the NGS technique to identify pathogenic and causative mutations, but also highlighted the crucial role of an expert team performing the critical revision of all detected WES variants.

\section{Acknowledgements}

The authors would like to thank the patient and his family for participating in this study. This work was supported by an Intramural grant of the Institute for Maternal and Child Health (IRCCS 'Burlo Garofolo', Trieste, Italy). The present study was also supported by the 'Associazione Azzurra Malattie Rare' and 'Beneficentia Stiftung in Vaduz, Liechtenstein'.

\section{References}

1. van Dijk EL, Auger H, Jaszczyszyn Y and Thermes C: Ten years of next-generation sequencing technology. Trends Genet 30: 418-426, 2014.

2. Pelak K, Shianna KV, Ge D, Maia JM, Zhu M, Smith JP, Cirulli ET, Fellay J, Dickson SP, Gumbs CE, et al: The characterization of twenty sequenced human genomes. PLoS Genet 6: e1001111, 2010

3. Robinson PN, Krawitz P and Mundlos S: Strategies for exome and genome sequence data analysis in disease gene discovery projects. Clin Genet 80: 127-132, 2011.

4. Danecek P, Auton A, Abecasis G, Albers CA, Banks E, DePristo MA, Handsaker RE, Lunter G, Marth GT, Sherry ST, et al; 1000 Genomes Project Analysis Group: The variant call format and VCF tools. Bioinformatics 27: 2156-2158, 2011.

5. Wang K, Li M and Hakonarson H: ANNOVAR: Functional annotation of genetic variants from high-throughput sequencing data. Nucleic Acids Res 38: e164, 2010

6. Adzhubei IA, Schmidt S, Peshkin L, Ramensky VE, Gerasimova A, Bork P, Kondrashov AS and Sunyaev SR: A method and server for predicting damaging missense mutations. Nat Methods 7 : 248-249, 2010.
7. Schwarz JM, Rödelsperger C, Schuelke M and Seelow D: MutationTaster evaluates disease-causing potential of sequence alterations. Nat Methods 7: 575-576, 2010.

8. Liu X, Jian X and Boerwinkle E: dbNSFP v2.0: A database of human non-synonymous SNVs and their functional predictions and annotations. Hum Mutat 34: E2393-E2402, 2013.

9. Tilley WD, Marcelli M, Wilson JD and McPhaul MJ: Characterization and expression of a cDNA encoding the human androgen receptor. Proc Natl Acad Sci USA: 86: 327-331, 1989.

10. Shoemaker JD and Elliott WH: Automated screening of urine samples for carbohydrates, organic and amino acids after treatment with urease. J Chromatogr A 562: 125-138, 1991.

11. Goldstein JL and Brown MS: Regulation of the mevalonate pathway. Nature 343: 425-430, 1990.

12. Haas D and Hoffmann GF: Mevalonate kinase deficiencies: From mevalonic aciduria to hyperimmunoglobulinemia D syndrome. Orphanet J Rare Dis 1: 13, 2006.

13. Bedoyan JK, Schaibley VM, Peng W, Bai Y, Mondal K, Shetty AC, Durham M, Micucci JA, Dhiraaj A, Skidmore JM, et al: Disruption of RAB40AL function leads to Martin-Probst syndrome, a rare X-linked multisystem neurodevelopmental human disorder. J Med Genet 49: 332-340, 2012.

14. Lee J, Wong S and Boles RG: Mutation in the X-linked RAB40AL gene (Martin-Probst syndrome) with mental retardation, sensorineural hearing loss, and anomalies of the craniofacies and genitourinary tract: A second case report. Eur J Pediatr 173: 967-969, 2014

15. Mandey SH, Schneiders MS, Koster J and Waterham HR: Mutational spectrum and genotype-phenotype correlations in mevalonate kinase deficiency. Hum Mutat 27: 796-802, 2006.

16. Marcuzzi A, Pontillo A, De Leo L, Tommasini A, Decorti G, Not T and Ventura A: Natural isoprenoids are able to reduce inflammation in a mouse model of mevalonate kinase deficiency. Pediatr Res 64: 177-182, 2008.

17. Ołdak M, Ścieżyńska A, Młynarski W, Borowiec M, Ruszkowska E, Szulborski K, Pollak A, Kosińska J, Mueller-Malesińska M, Stawiński P, et al: Evidence against RAB40AL being the locus for Martin-Probst X-linked deafness-intellectual disability syndrome. Hum Mutat 35: 1171-1174, 2014.

18. Kalscheuer V, Iqbal Z, Hu H, et al: RAB40AL loss-of-function mutation does not cause X-linked intellectual disability. J Med Genet: Reply to reference 1, 2013.

19. Allen RC, Zoghbi HY, Moseley AB, Rosenblatt HM and Belmont JW: Methylation of HpaII and HhaI sites near the polymorphic CAG repeat in the human androgen-receptor gene correlates with X chromosome inactivation. Am J Hum Genet 51: 1229-1239, 1992.

20. Wolf SF, Jolly DJ, Lunnen KD, Friedmann T and Migeon BR: Methylation of the hypoxanthine phosphoribosyltransferase locus on the human X chromosome: Implications for X-chromosome inactivation. Proc Natl Acad Sci USA 81: 2806-2810, 1984. 